OPEN ACCESS

Edited by:

Giacinto Bagetta,

University of Calabria, Italy

Reviewed by:

Kengo Hamamura,

Daiichi University of Pharmacy, Japan

Stefano Pieretti,

National Institute of Health, Italy

*Correspondence:

Roberta Lattanzi

roberta.lattanzi@uniroma1.it

Specialty section:

This article was submitted to

Neuropharmacology,

a section of the journal

Frontiers in Pharmacology

Received: 11 December 2020 Accepted: 28 January 2021

Published: 01 March 2021

Citation:

Lattanzi R, Severini C, Maftei D, Saso L and Badiani $A$ (2021) The Role of Prokineticin 2 in Oxidative Stress and in Neuropathological Processes. Front. Pharmacol. 12:640441. doi: 10.3389/fphar.2021.640441

\section{The Role of Prokineticin 2 in Oxidative Stress and in Neuropathological Processes}

\author{
Roberta Lattanzi ${ }^{1 *}$, Cinzia Severini ${ }^{2}$, Daniela Maftei ${ }^{1}$, Luciano Saso $^{1}$ and Aldo Badiani ${ }^{1}$ \\ ${ }^{1}$ Department of Physiology and Pharmacology "Vittorio Erspamer", Sapienza University of Rome, Rome, Italy, ${ }^{2}$ Institute of \\ Biochemistry and Cell Biology, IBBC, CNR, Rome, Italy
}

The prokineticin (PK) family, prokineticin 1 and Bv8/prokineticin 2 (PROK2), initially discovered as regulators of gastrointestinal motility, interacts with two G proteincoupled receptors, PKR1 and PKR2, regulating important biological functions such as circadian rhythms, metabolism, angiogenesis, neurogenesis, muscle contractility, hematopoiesis, immune response, reproduction and pain perception. PROK2 and $\mathrm{PK}$ receptors, in particular PKR2, are widespread distributed in the central nervous system, in both neurons and glial cells. The PROK2 expression levels can be increased by a series of pathological insults, such as hypoxia, reactive oxygen species, beta amyloid and excitotoxic glutamate. This suggests that the PK system, participating in different cellular processes that cause neuronal death, can be a key mediator in neurological/neurodegenerative diseases. While many PROK2/PKRs effects in physiological processes have been documented, their role in neuropathological conditions is not fully clarified, since PROK2 can have a double function in the mechanisms underlying to neurodegeneration or neuroprotection. Here, we briefly outline the latest findings on the modulation of PROK2 and its cognate receptors following different pathological insults, providing information about their opposite neurotoxic and neuroprotective role in different pathological conditions.

Keywords: neurotoxicity, neurodegenerative diseases, prokineticin receptors, Prokineticin receptor antagonists, prokineticin 2

\section{INTRODUCTION}

Prokineticins (PKs) represent a family of chemokine-like peptides characterized by their ability to induce hyperalgesia and gastrointestinal motility. The first PK was discovered about twenty years ago and named Bv8 to denote its origin, the skin secretions of Bombina variegata, and its molecular weight, $8 \mathrm{kDa}$ (Mollay et al., 1999). The family also includes MIT-1 (Mamba Intestinal Toxin 1) isolated from the venom of Black Mamba (Schweitz et al., 1999) and the mammalian Prokineticin 1 (PROK1) and Prokineticin 2 (PROK2) (Li et al., 2001).

PKs structure consists of a highly conserved hexapeptide in N-terminal position and ten cysteine residues, that form five disulphide bridges, giving to the molecule a very compact structure (Negri and Ferrara, 2018). This represents a substantial difference compared to that of chemokines characterized, instead, by four to six cysteine residues. 
TABLE 1 | PK system modulation related to neurotoxic or neuroprotective activity following different pathological insults.

Pathological insult PROK2 activity Results

Oxidative stress Neurotoxic $\quad$-Primary cortical cell cultures

-in vivo stroke models

Neuroprotective -in vitro model of focal cerebral ischemia

Beta amyloid Neurotoxic $\quad$-Primary cortical cell cultures

-Tg2576 transgenic mouse model of AD $\downarrow$

-in vivo non-transgenic rat model of $A D$

$-A D$ patients

Glutamate

Neurotoxic

-Primary cortical cell cultures

-Primary striatal cell cultures

Neuroprotective -Primary cortical cell cultures

-Primary mouse astrocytes

-in vitro model of ischemic tolerance

Dopaminergic neurons toxicity
Neuroprotective $\quad$-N27 dopaminergic neuronal cell cultures

-Primary mesencephalic neuronal cell cultures

-in vivo MPTP model of PD
-MitoPark mice transgenic model of PD

-PD patients
-Hypoxia and ROS: $\uparrow$ PROK2 mRNA mainly in neurons (Cheng et al., 2012) -Ischemic cortex and striatum: $\uparrow$ PROK2 mRNA (Cheng et al., 2012) -PROK2 i.c.v. injection after stroke: $\uparrow$ infarct volume (Cheng et al., 2012) - PKRs antagonist: $\downarrow$ infarct volume, $\downarrow$ central inflammation and improves behavioral outcome (Cheng et al., 2012)

-Bv8: $\downarrow$ the OGD-induced necrotic neuronal death in cortical cultures and hippocampal slices activating ERK, Akt and GSK3- $\beta$ pathways (Landucci et al. 2016)

-PKRs antagonist reverts the Bv8 effects (Landucci et al., 2016)

-Hippocampal slices: $\uparrow$ PROK2, PKR1 and PKR2 mRNA and protein (Landucci et al., 2016)

-PKRs antagonist: $\downarrow$ PROK2 protein in hippocampal slices (Landucci et al., 2016)

-PROK2 treatment or PKR1 overexpression: $\downarrow$ oxidative stress-induced apoptotic cell death activating the Akt/mTOR and AKT/GSK3 $\beta$ pathways (Urayama et al., 2007; Su et al., 2020; Yang et al., 2020)

$-A \beta_{1-42}: \uparrow P R O K 2, P K R 1$ and PKR2 mRNA and protein levels in neurons and astrocytes (Severini et al., 2015)

-Bv8, at pM concentrations, $\uparrow$ neuronal apoptosis (Severini et al., 2015)

-PKRs antagonist: $\downarrow A \beta$ - and Bv8-induced neuronal apoptosis (Severini et al.,

2015) and $\downarrow$ kainate-evoked current increase $A \beta$ - induced (Caioli et al., 2017)

-Cortex and hippocampus: $\uparrow$ PROK2 mRNA (Lattanzi et al., 2019)

-PKRs antagonist: $\downarrow$ LTP impairment in hippocampal slices (Severini et al., 2015)

$-A \beta_{1-42}$ i.c.v. injection: $\uparrow$ PROK2, PKR1 and PKR2 mRNA and protein in

prefrontal cortex and hippocampus (Severini et al., 2015; Lattanzi et al., 2019; Maftei et al., 2019)

-PKRs antagonist: $\downarrow$ PROK2 levels, $\downarrow$ glial activation and neuronal death,

restores the neurogenesis in DG and $\downarrow$ the $A \beta$-induced memory deficits (Maftei et al., 2019)

- $\uparrow$ PROK2 mRNA in hippocampus and $\uparrow$ PROK2 protein in serum (Lattanzi

et al., 2019)

-NMDA and excitotoxic glutamate: $\uparrow$ PROK2 mRNA mainly in neurons (Cheng

et al., 2012)

-Bv8, at pM concentrations, $\uparrow$ AMPA currents (Caioli et al., 2017)

-PKRs antagonist protects neurons from kainate-induced death (Caioli et al., 2017)

-Excitotoxic glutamate: $\uparrow$ PROK2 mRNA (Cheng et al., 2012)

-Bv8, at $\mu \mathrm{M}$ concentrations protects the cortical neurons against NMDA toxicity

(Melchiorri et al., 2001)

-PROK2 and IS20 (a PKR1 agonist): $\uparrow$ astrocytes uptake of extracellular

glutamate (Neal et al., 2018)

-NMDA-induced OGD tolerance: $\uparrow$ PROK2/PKRs protein (Landucci et al., 2016)

-PKRs antagonist: $\downarrow$ PROK2 and $\downarrow$ the development of NMDA-induced OGD tolerance and blocks the NMDA-induced activation of ERK1/2 and Akt (Landucci et al., 2016)

$-\mathrm{MPP}^{+}$and TNFa: $\uparrow$ PROK2 mRNA and protein in DAergic neurons (Gordon et al., 2016)

-PROK2: Protects against MPP ${ }^{+}$-mediated oxidative stress, mitochondrial dysfunction and neuronal cell death activating ERK and Akt pathways (Gordon et al., 2016)

-PROK2: $\downarrow \mathrm{MPP}^{+}$-induced DAergic neuronal loss (Gordon et al., 2016)

-DAergic neurons of nigral tissue: $\uparrow$ PROK2 protein (Gordon et al., 2016) -PROK2: $\downarrow$ MPTP-induced behavioral deficits and DAergic degeneration (Gordon et al., 2016)

-PKRs antagonist: $\uparrow$ DAergic degeneration and $\uparrow$ the behavioral deficits (Gordon et al., 2016)

-IS20: Protects against MPTP-induced reduction of astrocytic A2

antiinflammatory phenotype and $\downarrow$ expression of proinflammatory factors (Neal et al., 2018)

-Nigral DAergic neurons: $\uparrow$ PROK2 following both acute and progressive

DAergic degeneration (Gordon et al., 2016)

-Nigral DAergic neurons: $\uparrow$ PROK2 protein (Gordon et al., 2016) 
PKs effects are mediated by biding with two $G$ protein-coupled membrane receptors: prokineticin receptor 1 (PKR1) and 2 (PKR2) which share an $85 \%$ identity in the amino acid sequence (Lin et al., 2002; Soga et al., 2002; Negri et al., 2009).

PKs and PKRs are present in a multiplicity of organs and tissues, including the central nervous system (CNS), gonads, adrenal gland, placenta, uterus, gastro-intestinal tract, heart, bone marrow and blood. Not surprisingly, the PKs have been shown to participate in a bewildering variety of physiological actions comprehending the coordination of complex behaviors as circadian rhythms, muscle contractility, reproduction, angiogenesis, neurogenesis, haematopoiesis, immune responses and pain perception (Negri and Ferrara, 2018).

Of particular relevance to the topic of this review, is the extended distribution of $\mathrm{PKs}$ and their receptors in the mammalian organs and tissues. In CNS the ligands are less expressed than the receptors. PROK1 is mainly present in the tractus solitarius and in the cerebellum. PROK2 is evident in several forebrain regions, mainly in the hypothalamus and in the suprachiasmatic nucleus (Cheng et al., 2002; Cheng et al., 2006), and in the spinal cord (Maftei et al., 2014). Regarding the receptors, their relative density in the brain changes as a function of discrete areas, but in most regions PKR2 is expressed more than PKR1 (Cheng et al., 2006; Negri and Ferrara, 2018). From a neurodevelopmental point of view, both PKR1 and PKR2 can be detected as early as at embryonic day 7 in mouse and in rat (Negri et al., 2007). In rat embryos, both PKR1 and PKR2 are highly expressed in the neuroepithelium lining the ventricles, olfactory bulb, Gasserganglion and Dorsal Root Ganglia (DRG). However, in neonatal and adult rat brain only PKR2 is still expressed at high levels in multiple areas, whereas PKR1 is detected at low density only in the cortex (Cheng et al., 2006; Negri et al., 2007; Zhang et al., 2009). PKR1 and particularly PKR2 are also present in the dorsal horns of the spinal cord and in DRG, indicating their role in the transmission of noxious stimuli (Maftei et al., 2014; Negri and Maftei, 2018).

The distribution of PROK2 and PKRs in the CNS varies as a function of cellular types. Studies conducted in primary cultures of neurons, astrocytes and microglia, obtained from embryo or neonate brain mice, have shown that PKR2 is mostly expressed in neurons, whereas PROK2 and PKR1 are mainly expressed in astrocytes and microglia (Koyama et al., 2006; Severini et al., 2015).

The widespread distribution of PROK2 and its receptors, mainly PKR2, in the CNS underlies not only their role in physiological functions, but also their participation in neuropathological processes. Indeed, PROK2 may be overexpressed following a series of pathological insults, such as hypoxia, reactive oxygen species (ROS), amyloid $\beta(\mathrm{A} \beta)$ and excitotoxic glutamate, suggesting its involvement in numerous processes that lead to neuronal death. Interestingly, in diverse neuronal populations and in different pathological conditions PROK2 can show both neurotoxic and neuroprotective effects (Table 1). We will focus on the response of the PK system to a variety of pathological insults and on the mechanisms implicated in its neurotoxic and neuroprotective effects.

\section{PKS INVOLVEMENT IN THE RESPONSE TO PATHOLOGICAL INSULTS}

\section{Oxidative Stress}

Oxidative stress indicates that cells suffer the harmful effects induced by excessive levels of ROS, potentially leading to damage to lipids, proteins or nucleic acids. Oxidative DNA damage occurs immediately after ischemic stroke and is generally reversible (Cui et al., 2000; Lan et al., 2003). This last point appears particularly interesting because it offers the possibility of a therapeutic treatment.

It has been reported (Cheng et al., 2012) that PROK2 mRNA levels increase in vitro, in primary cortical cultures following hypoxia and ROS treatment, and also in an in vivo model of stroke obtained by transient intraluminal middle cerebral artery occlusion. The PROK2 increase was observed in the ischemic cortex and striatum and was proportionally to the severity of damage, indicating that the stroke-induced PROK2 up-regulation is a consequence of the ischemic damage. The PROK2 overexpression proved to be harmful since the exogenous PROK2 post-stroke delivery worsened the ischemic injury, whereas blocking PROK2 expression reduced the infarct volume and central inflammation and improved behavioral outcome, suggesting that impairing the PROK2 levels could be therapeutic The harmful effect of PROK2 is also supported by the signal transduction pathways activation. Indeed, treatment of primary neuron cultures with PROK2, after exposure to glutamate excitotoxicity or oxygen glucose deprivation (OGD), increased both pERK1,2 and pSAP/JNK MAPK levels without changes in pAkt levels, indicating that these pathways are partially responsible for PROK2-induced deleterious effects in ischemic injury. Although ERK1,2 and Akt signaling exert mostly beneficial effects (Zhao et al., 2005; Zhao et al., 2006), they also may be involved in deleterious effects, increasing ischemic damage (Sawe et al., 2008). On the whole, the authors suggested that PROK2 is an endangering mediator for ischemic brain injury and a compelling target for stroke treatment.

Conversely, a neuroprotective role for PROK2 has been also reported in cerebral ischemia (Landucci et al., 2016). In this study, Bv8 (the amphibian PROK2 homologue) exerted neuroprotective effect in two in vitro models of cerebral ischemia, cortical cell cultures and in organotypic hippocampal slices exposed to OGD. Furthermore, the ischemic tolerance, induced by the exposure of hippocampal slices to a preconditioning sub-toxic N-methyl-Daspartate (NMDA) stimulus before the exposure to OGD (Gerace et al., 2012), led to an increase of PROK2/PKRs mRNA and protein levels which was inhibited by PC7, a PKRs antagonist. The PROK2 neuroprotective effect was associated to the activation of the ERK1/2 and Akt transduction pathways (Landucci et al., 2016).

A possible explanation for the discrepancy between neuroprotective and neurotoxic effects exerted by PROK2, in vitro, could be ascribed to the different range of concentrations used. Protective/pro-survival effect of PROK2 was generally obtained with nanomolar concentrations of Bv8/PROK2, 

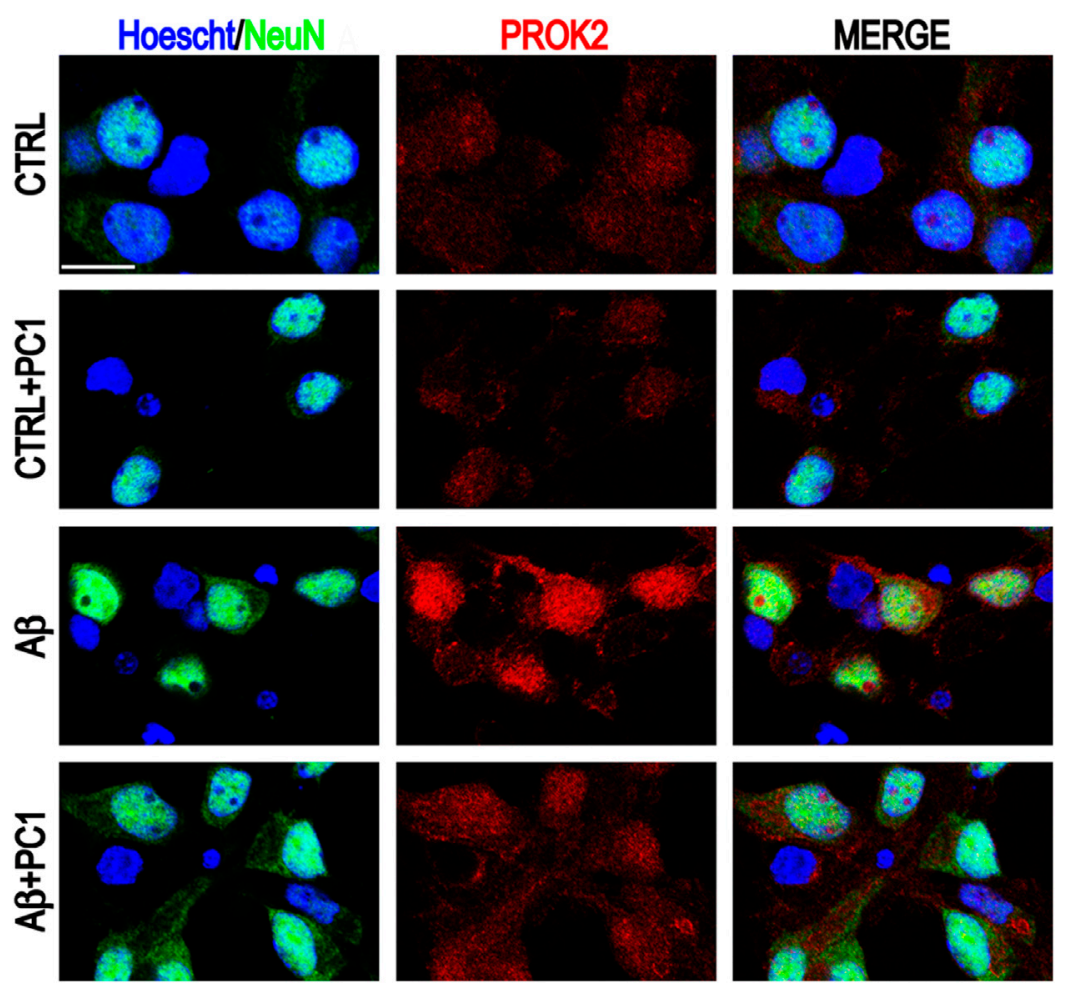

FIGURE 1 | Representative immunofluorescence images of cultured mixed cortical neurons (CNs) stained with anti-PROK2 antibody (red) in control conditions (CTRL) and after 48 h of $A \beta_{1-42}$ treatment alone or in the presence of PC1 (200 nM). Neurons were stained with NeuN (green), and nuclei with Hoecsht (blue). Scale bar: $15 \mu \mathrm{m}$ (Severini et al., 2015).

able to activate the pro-survival ERK1/2 and Akt transduction pathways (Melchiorri et al., 2001; Negri et al., 2005; Ng et al., 2005; Urayama et al., 2008; Gasser et al., 2015). Conversely, the proapoptotic effect of PROK2 was obtained at picomolar concentrations, acting on the SAP/JNK pathway under excitotoxic or OGD conditions (Cheng et al., 2012; Gasser et al., 2015; Severini et al., 2015).

Protective effects of PROK2 were also demonstrated in other organ systems. PROK2, through PKR1 binding and PKR1 overexpression, protected the cardiomyocytes from oxidative stress-induced apoptosis through Akt pathway activation. These effects were completely nullified by siRNA-PKR1. In the same work, in an in vivo animal model of myocardial infarction, induced by coronary ligation, the authors demonstrated that PKR1 gene therapy preserved the myocardial function by blocking the apoptosis and stimulating angiogenesis (Urayama et al., 2007; Urayama et al., 2008). In line with this data, recent studies have confirmed that PROK2 exerts its protective role on cardiomyocytes by activating the Akt/mTOR (Su et al., 2020) and AKT/GSK3 $\beta$ (Yang et al., 2020) signaling pathways.

\section{A $\beta$ Toxicity}

The presence of extracellular $A \beta$ plaques, tau neurofibrillary tangles and gliosis are associated with the pathogenesis of Alzheimer's disease (AD) (Akiyama et al., 2000; Crews and Masliah, 2010). Activated astrocytes and microglia are the main source of pro-inflammatory factors: among them, chemokines and chemokine receptors have been shown to be over-expressed in AD brain (Rubio-Perez and Morillas-Ruiz, 2012; Zuena et al., 2019). The new chemokine-like peptide PROK2 and PKRs were found to be modulated in response to $\mathrm{A} \beta$ insult. Indeed, in mixed primary rat cortical cultures and in cortex and hippocampus from A $\beta$-treated rats, PROK2, PKR1, and PKR2 mRNA and protein levels were significantly upregulated by $\mathrm{A} \beta$ treatment (Severini et al., 2015; Caioli et al., 2017). PROK2 was significantly increased in astrocytes, the principal cells producing chemokines after $A \beta$ insult (Johnstone et al., 1999; Severini et al., 2013) and in neurons; PKR1 was mainly increased in neurons while PKR2 in both neurons and astrocytes (Severini et al., 2015).

The neurotoxic/deleterious activity of the PK system in $\mathrm{A} \beta$ induced neurotoxicity was further demonstrated by the ability of PC1, a PKRs antagonist (Balboni et al., 2008; Congiu et al., 2014; Lattanzi et al., 2014), to concentration-dependently prevent A $\beta$ induced apoptosis and to reduce the PROK2 increase. Indeed, the exposure of primary cortical cultures to $A \beta_{1-42}$ strongly induced PROK2 overexpression in neurons that was significantly decreased by PC1 treatment (Figure 1), impairing the proapoptotic signaling and confirming previous data which demonstrate the PC1 ability to reduce PROK2 expression and storage in mice spinal neurons and astrocytes following peripheral nerve injury (Maftei et al., 2014; Lattanzi et al., 2015). 
The neurotoxic role of PROK2 in A $\beta$ toxicity is supported also by the Bv8 ability to induce in cortical brain cultures, at picomolar concentrations, apoptosis comparable to that exerted by $\mathrm{A} \beta$, pointing out that such a low concentrations could be in accordance with the small amount of PROK2 eventually released by the $A \beta$ treatment. The Bv8-induced apoptosis was prevented by co-administration of $\mathrm{PC} 1$, which prevented also the long-term potentiation (LTP) impairment in hippocampal slices of Tg2576 transgenic mice compared with age-matched wild-type controls (Severini et al., 2015). The PC1 capability to antagonize A $\beta$ toxicity and LTP impairment in the Tg2576 transgenic mice, indicates that damage-induced PROK2 overexpression is deleterious and that blocking the PK system may represent a therapeutic strategy.

In another study it was reported that, in neurons from primary cortical cultures, PC1 reverted the increase of ionic current induced by $A \beta$ trough the AMPA ( $\alpha$-amino-3-hydroxy-5methyl-4-isoxazole-propionic acid) receptors and protected the cortical neurons against kainite-induced death. Interestingly, still in cortical neurons, picomolar concentrations of Bv8 increased both evoked and spontaneous AMPA currents and this effect was nullified by PC1.

The Bv8 effect was not due to an increase of AMPA receptor subunit expression. Instead, it seems to be mediated by activation of the intracellular protein kinase $\mathrm{C}$ (PKC) since the $\mathrm{PKC}$ inhibitor Go6983 counteracted the effect (Caioli et al., 2017).

A recent paper highlights a neuroprotective role of $\mathrm{PC} 1$ on $\mathrm{A} \beta$-induced memory impairment in rats through the modulation of the PK system. A $\beta$ intracerebroventricular (i.c.v) infusion induced a strong cognitive dysfunction and a significant up-regulation of PROK2 and PKRs in cortical and hippocampal neurons and astrocytes. Subcutaneous treatment with PC1 prevented the A $\beta$-induced memory reduction restoring not only the balance of the whole $\mathrm{PK}$ system but also the physiological neurogenesis in Dentate Gyrus (Maftei et al., 2019) which results compromised in AD brains (Li et al., 2008). Moreover, a significant PROK2 upregulation was also demonstrated in brain of $\mathrm{Tg} 2576$ transgenic $\mathrm{AD}$ mice and in both brain and serum of $\mathrm{AD}$ patients (Lattanzi et al., 2019).

The PK system seems to be a key mediator in the A $\beta$-induced neuronal damage and PK antagonists may represent new therapeutic drugs to ameliorate the $\mathrm{AD}$ progression. The significant PROK2 increase in $\mathrm{AD}$ patient's serum could indicate PROK2 as a potential blood-based biomarker of the pathology.

\section{Glutamatergic Toxicity}

A growing body of evidence suggests that glutamate receptormediating excitotoxicity plays a pivotal role in the pathogenesis of many neurodegenerative diseases such as AD, Huntington's, Parkinson's and Amyotrophic Lateral Sclerosis disease (Lipton and Rosemberg, 1994; Blandini et al., 1996).

The exposure of primary cortical cultures to excitotoxic glutamate and NMDA induced PROK2 mRNA overexpression. The glutamate-induced PROK2 up-regulation depended by NMDA receptor activation, required extracellular
$\mathrm{Ca}^{2+}$ and was present mainly in neurons (Cheng et al., 2012). Furthermore Bv8 increased, in cortical neurons, the AMPA currents evoked by kainate perfusion and its effect was annulled by PC1 (Caioli et al., 2017), indicating that a dysregulation of the PK system balance may have a deleterious role in glutamatergic neurotoxicity.

However, the harmful activity of Bv8 in glutamate neurotoxicity disagrees with previously reported data which demonstrated that, in murine cortical cultures, Bv8 protected against NMDA-induced excitotoxicity through the activation of the MAPK and PI3-K pathways (Melchiorri et al., 2001). In support of these data, other recent studies reported a protective role of PROK2 in a model of ischemic tolerance in vitro, induced by the pre-exposure of hippocampal slices to a preconditioning subtoxic concentration of NMDA before the exposure to OGD. Indeed, the block of PKRs prevented the development of the NMDA-induced OGD tolerance and the subsequent PROK2 increase, and blocked the activation of the pro-survival ERK1/2 and PI-3 kinase/Akt signaling pathways normally promoted by the NMDA preconditioning stimulus (Landucci et al., 2016).

In another study, Neal and colleagues (Neal et al., 2018) suggested that the neuroprotective role of PROK2 may be due also to its ability to increase the uptake of extracellular glutamate by astrocytes through the upregulation of the glutamate transporter GLAST. Taken together, these data suggest that PROK2/PKRs signaling may play an important role also in molecular mechanisms underlying neuroprotection.

\section{Dopaminergic Neurons Toxicity}

The degeneration and the progressive loss of the dopaminergic (DA) neurons in the substantia nigra pars compacta (SNpc) is considered the most important hallmark of Parkinson's disease (PD).

The involvement of PROK2 in dopaminergic neurodegeneration was proposed since its expression is strongly increased, both in vitro and in vivo, in DA neurons during the neurodegeneration process. In vitro, the exposure of the dopaminergic neurons to the TNFa (tumor necrosis factor alpha) or parkinsonian neurotoxin 1-methyl-4phenylpyridinium $\left(\mathrm{MPP}^{+}\right)$, important contributors to the pathogenesis of $\mathrm{PD}$, increased the expression of both PROK2 mRNA and secreted protein in the early stage of neuronal death (Gordon et al., 2016). In agreement, in vivo data from preclinical mouse models of PD, MPTP model (Jackson-Lewis and Przedborski, 2007) and transgenic MitoPark mice (Ekstrand and Galter, 2009), indicated an early PROK2 upregulation in nigral DA neurons before the onset of neuronal degeneration and motor deficits. These data indicate that PROK2 is an inducible secreted mediator upregulated precociously in the DA neurons in response to neurotoxic stress. In addition, PROK2 protein expression was found elevated also in nigral tissue (Gordon et al., 2016) and in serum from PD patients (Schirinzi et al., 2021). PROK2 serum increase correlates with CSF A $\beta$ increase and lactate decrease, suggesting that PROK2 may have a protective role at synaptic level and an antioxidant action promoting recovery from mitochondrial damage. 
Regarding PKR2, its constitutive expression in SN remained unaltered in both MPTP-treated mice and in PD patients' brain (Gordon et al., 2016).

PROK2 overexpression in DA neurons has a neuroprotective role since its administration, via selective interaction with the cognate PKR2, reduced the $\mathrm{MPP}^{+}$-induced neuronal cell death, oxidative stress and mitochondrial dysfunction and promoted the mitochondrial biogenesis in dopaminergic neurons. PROK2 exerted its protective effects through the activation of ERK and Akt signaling pathways, and the upregulation of PGC-1 $\alpha$ and TFAM, the main mediators of mitochondrial biogenesis (Gordon et al., 2016). The protective effect of PROK2 could be also associated to its ability to induce an anti-inflammatory A2 phenotype in astrocytes. Indeed, in astrocytes cultures, thought selective interaction with the PKR1, PROK2 reduced the levels of ROS and pro-inflammatory factors, such as TNFa and IL1 $\beta$, increased the astrocytic uptake of glutamate and the expression of neuroprotective factors such as the antioxidant arginase-1 or Nrf2 (Neal et al., 2018). Nrf2 overexpression in astrocytes, as well as the astrocytic ability to remove the extracellular glutamate, has been demonstrated to protect the dopaminergic neurons from death (Yao et al., 2005; Gan et al., 2012).

Moreover, PROK2 administration into the mouse striatum, significantly improved the MPTP-induced locomotor deficits and protected against dopaminergic neuronal degeneration, while the administration PKRA7, a PKRs antagonist, blocked the protective function of PROK2 (Gordon et al., 2016; Neal et al., 2018).

Since glial activation has been reported in many neurodegenerative disorders, including $\mathrm{PD}$, and this activation contribute to the disease progression (Guzman-Martinez et al., 2019; Li et al., 2019), the upregulation of PROK2 in dopaminergic neurons represents not only a local protective mechanism against neuronal death but could also play an important role in neuronglia cross-talk. Given the neuroprotective activity of PROK2, it is possible to speculate that agonists for PK receptors might represent a novel neuroprotective therapeutic approach that can slow down or halt the dopaminergic neuronal degeneration in PD.

\section{REFERENCES}

Akiyama, H., Barger, S., Barnum, S., Bradt, B., Bauer, J., Cole, G. M., et al. (2000). Inflammation and Alzheimer's disease. Neurobiol. Aging 21 (3), 383-421. doi:10.1016/s0197-4580(00)00124-x

Balboni, G., Lazzari, I., Trapella, C., Negri, L., Lattanzi, R., Giannini, E., et al. (2008). Triazine compounds as antagonists at Bv8-prokineticin receptors. J. Med. Chem. 51 (23), 7635-7639. doi:10.1021/jm800854e

Blandini, F., Porter, R. H., and Greenamyre, J. T. (1996). Glutamate and Parkinson's disease. Mol. Neurobiol. 12 (1), 73-94. doi:10.1007/BF02740748

Caioli, S., Severini, C., Ciotti, T., Florenzano, F., Pimpinella, D., Petrocchi Passeri, P., et al. (2017). Prokineticin system modulation as a new target to counteract the amyloid beta toxicity induced by glutamatergic alterations in an in vitro model of Alzheimer's disease. Neuropharmacology 116 (2017), 82-97. doi:10. 1016/j.neuropharm.2016.12.012

Cheng, M. Y., Bullock, C. M., Li, C., Lee, A. G., Bermak, J. C., Belluzzi, J., et al. (2002). Prokineticin 2 transmits the behavioural circadian rhythm of the suprachiasmatic nucleus. Nature 417 (6887), 405-410. doi:10.1038/ $417405 \mathrm{a}$

\section{CONCLUSIONS}

The data presented in this review bring evidence that activation of the PK system by various pathological stressors can exert both neuroprotective and neurotoxic effects in diverse experimental conditions and human pathologies. These studies highlight that PROK2 neuronal activity depends on various factors including the specific neuronal population, the amount of PROK2 released and the type of receptor activated. Depending on the amount released by pathological insults (in the range of pmol or nmol) PROK2 could activate a different fraction of PKR1 and PKR2 and different, sometimes opposite, transduction pathways. In the CNS, the proper role of PKR1 and PKR2 regarding the mechanisms leading to neuroprotection or neurodegeneration has not been fully understood yet, mainly for the absence of specific PK receptor subtype antagonists. The PROK2 pro-survival/ neuroprotective effects at nanomolar concentrations range requires mainly the activation of ERK1/2 and Akt signaling pathways, whereas the damaging effects of PKs picomolar concentrations seems to involve ERK1,2 and SAP/JNK pathway, without involvement of Akt signaling pathways.

Thus, further studies are needed to clarify how the receptordependent signaling can modulate PROK2 responses by analyzing PKR1-or PKR2-deficient mice with different treatments that induce neuroprotection or neurodegeneration. Understanding the role of PROK2 and its receptors in neuronal death and/or survival may be a crucial step in clarifying the mechanisms involved in various neuropathologies.

\section{AUTHOR CONTRIBUTIONS}

RL and CS developed the manuscript, searched the literature and wrote the manuscript, DM prepared the table and the figure, $\mathrm{AB}$ and LS corrected the style, reviewed and edited the manuscript. All authors have read and approved the final manuscript.

Cheng, M. Y., Lee, A. G., Culbertson, C., Sun, G., Talati, R. K., Manley, N. C., et al. (2012). Prokineticin 2 is an endangering mediator of cerebral ischemic injury. Proc. Natl. Acad. Sci. USA 109 (14), 5475-5480. doi:10.1073/pnas.1113363109

Cheng, M. Y., Leslie, F. M., and Zhou, Q. Y. (2006). Expression of prokineticins and their receptors in the adult mouse brain. J. Comp. Neurol. 498 (6), 796-809. doi: $10.1002 / \mathrm{cne} .21087$

Congiu, C., Onnis, V., Deplano, A., Salvadori, S., Marconi, V., Maftei, D., et al. (2014). A new convenient synthetic method and preliminary pharmacological characterization of triazinediones as prokineticin receptor antagonists. Eur. J. Med. Chem. 81, 334-340. doi:10.1016/j.ejmech.2014.05.030

Crews, L., and Masliah, E. (2010). Molecular mechanisms of neurodegeneration in Alzheimer's disease. Hum. Mol. Genet. 19 (R1), R12-R20. doi:10.1093/hmg/ ddq160

Cui, J., Holmes, E. H., Greene, T. G., and Liu, P. K. (2000). Oxidative DNA damage precedes DNA fragmentation after experimental stroke in rat brain. FASEB J. 14 (7), 955. doi:10.1096/fasebj.14.7.955

Ekstrand, M. I., and Galter, D. (2009). The MitoPark Mouse - an animal model of Parkinson's disease with impaired respiratory chain function in dopamine neurons. Parkinsonism Relat. Disord. 15 Suppl 3 (3), S185-S188. doi:10.1016/ S1353-8020(09)70811-9 
Gan, L., Vargas, M. R., Johnson, D. A., and Johnson, J. A. (2012). Astrocytespecific overexpression of Nrf2 delays motor pathology and synuclein aggregation throughout the CNS in the alpha-synuclein mutant (A53T) mouse model. J. Neurosci. 32 (49), 17775-17787. doi:10.1523/ JNEUROSCI.3049-12.2012

Gasser, A., Brogi, S., Urayama, K., Nishi, T., Kurose, H., Tafi, A., et al. (2015). Discovery and cardioprotective effects of the first non-Peptide agonists of the $\mathrm{G}$ protein-coupled prokineticin receptor-1. PLoS One 10 (4), e0121027. doi:10. 1371/journal.pone. 0121027

Gerace, E., Scartabelli, T., Formentini, L., Landucci, E., Moroni, F., Chiarugi, A., et al. (2012). Mild activation of poly(ADP-ribose) polymerase (PARP) is neuroprotective in rat hippocampal slice models of ischemic tolerance. Eur. J. Neurosci. 36 (1), 1993-2005. doi:10.1111/j.1460-9568.2012.08116.x

Gordon, R., Neal, M. L., Luo, J., Langley, M. R., Harischandra, D. S., Panicker, N., et al. (2016). Prokineticin-2 upregulation during neuronal injury mediates a compensatory protective response against dopaminergic neuronal degeneration. Nat. Commun. 7, 12932. doi:10.1038/ncomms12932

Guzman-Martinez, L., Maccioni, R. B., Andrade, V., Navarrete, L. P., Pastor, M. G., and Ramos-Escobar, N. (2019). Neuroinflammation as a common feature of neurodegenerative disorders. Front. Pharmacol. 10, 1008. doi:10.3389/fphar. 2019.01008

Jackson-Lewis, V., and Przedborski, S. (2007). Protocol for the MPTP mouse model of Parkinson's disease. Nat. Protoc. 2 (1), 141-151. doi:10.1038/nprot.2006.342

Johnstone, M., Gearing, A. J., and Miller, K. M. (1999). A central role for astrocytes in the inflammatory response to beta-amyloid; chemokines, cytokines and reactive oxygen species are produced. J. Neuroimmunol. 93 (1-2), 182-193. doi:10.1016/s0165-5728(98)00226-4

Koyama, Y., Kiyo-oka, M., Osakada, M., Horiguchi, N., Shintani, N., Ago, Y., et al. (2006). Expression of prokineticin receptors in mouse cultured astrocytes and involvement in cell proliferation. Brain Res. 1112 (1), 65-69. doi:10.1016/j. brainres.2006.07.013

Lan, J., Li, W., Zhang, F., Sun, F. Y., Nagayama, T., O’Horo, C., et al. (2003). Inducible repair of oxidative DNA lesions in the rat brain after transient focal ischemia and reperfusion. J. Cereb. Blood Flow Metab. 23 (11), 1324-1339. doi:10.1097/01.WCB.0000091540.60196.F2

Landucci, E., Lattanzi, R., Gerace, E., Scartabelli, T., Balboni, G., Negri, L., et al. (2016). Prokineticins are neuroprotective in models of cerebral ischemia and ischemic tolerance in vitro. Neuropharmacology 108, 39-48. doi:10.1016/j. neuropharm.2016.04.043

Lattanzi, R., Congiu, C., Onnis, V., Deplano, A., Salvadori, S., Marconi, V., et al. (2014). Halogenated triazinediones behave as antagonists of pkr1: in-vitro and in-vivo pharmacological characterization. Intern. J. Pharmaceut. Scie. Res. 5, 5066-5074. doi:10.13040/IJPSR.0975-8232.6(3).1033-42

Lattanzi, R., Maftei, D., Marconi, V., Florenzano, F., Franchi, S., Borsani, E., et al. (2015). Prokineticin 2 upregulation in the peripheral nervous system has a major role in triggering and maintaining neuropathic pain in the chronic constriction injury model. Biomed. Res. Int. 2015, 301292. doi:10.1155/2015/ 301292

Lattanzi, R., Maftei, D., Petrella, C., Pieri, M., Sancesario, G., Schirinzi, T., et al. (2019). Involvement of the chemokine prokineticin-2 (PROK2) in alzheimer's disease: from animal models to the human pathology. Cells 8, 1430. doi:10.3390/cells8111430

Li, B., Yamamori, H., Tatebayashi, Y., Shafit-Zagardo, B., Tanimukai, H., Chen, S., et al. (2008). Failure of neuronal maturation in Alzheimer disease dentate Gyrus. J. Neuropathol. Exp. Neurol. 67 (1), 78-84. doi:10.1097/nen.0b013e318160c5db

$\mathrm{Li}, \mathrm{K}$., Li, J., Zheng, J., and Qin, S. (2019). Reactive astrocytes in neurodegenerative diseases. Aging Dis. 10 (3), 664-675. doi:10.14336/AD.2018.0720

Li, M., Bullock, C. M., Knauer, D. J., Ehlert, F. J., and Zhou, Q. Y. (2001). Identification of two prokineticin cDNAs: recombinant proteins potently contract gastrointestinal smooth muscle. Mol. Pharmacol. 59 (4), 692-698. doi:10.1124/mol.59.4.692

Lin, D. C., Bullock, C. M., Ehlert, F. J., Chen, J. L., Tian, H., and Zhou, Q. Y. (2002). Identification and molecular characterization of two closely related $\mathrm{G}$ proteincoupled receptors activated by prokineticins/endocrine gland vascular endothelial growth factor. J. Biol. Chem. 277 (22), 19276-19280. doi:10.1074/jbc.M202139200

Lipton, S. A., and Rosenberg, P. A. (1994). Excitatory amino acids as a final common pathway for neurologic disorders. N. Engl. J. Med. 330 (9), 613-622. doi:10.1056/NEJM199403033300907
Maftei, D., Marconi, V., Florenzano, F., Giancotti, L. A., Castelli, M., Moretti, S., et al. (2014). Controlling the activation of the Bv8/prokineticin system reduces neuroinflammation and abolishes thermal and tactile hyperalgesia in neuropathic animals. Br. J. Pharmacol. 171 (21), 4850-4865. doi:10.1111/ bph.12793

Maftei, D., Ratano, P., Fusco, I., Marconi, V., Squillace, S., Negri, L., et al. (2019). The prokineticin receptor antagonist $\mathrm{PC} 1$ rescues memory impairment induced by $\beta$ amyloid administration through the modulation of prokineticin system. Neuropharmacology 158, 107739. doi:10.1016/j.neuropharm.2019.107739

Melchiorri, D., Bruno, V., Besong, G., Ngomba, R. T., Cuomo, L., De Blasi, A., et al. (2001). The mammalian homologue of the novel peptide Bv8 is expressed in the central nervous system and supports neuronal survival by activating the MAP kinase/PI-3-kinase pathways. Eur. J. Neurosci. 13 (9), 1694-1702. doi:10.1046/j. 1460-9568.2001.01549.x

Mollay, C., Wechselberger, C., Mignogna, G., Negri, L., Melchiorri, P., Barra, D., et al. (1999). Bv8, a small protein from frog skin and its homologue from snake venom induce hyperalgesia in rats. Eur. J. Pharmacol. 374 (2), 189-196. doi:10. 1016/s0014-2999(99)00229-0

Neal, M., Luo, J., Harischandra, D. S., Gordon, R., Sarkar, S., Jin, H., et al. (2018). Prokineticin-2 promotes chemotaxis and alternative A2 reactivity of astrocytes. Glia 66 (10), 2137-2157. doi:10.1002/glia.23467

Negri, L., and Ferrara, N. (2018). The prokineticins: neuromodulators and mediators of inflammation and myeloid cell-dependent angiogenesis. Physiol. Rev. 98 (2), 1055-1082. doi:10.1152/physrev.00012.2017

Negri, L., Lattanzi, R., Giannini, E., Canestrelli, M., Nicotra, A., and Melchiorri, P. (2009). Bv8/Prokineticins and their receptors A new pronociceptive system. Int. Rev. Neurobiol. 85, 145-157. doi:10.1016/S0074-7742(09)85011-3

Negri, L., Lattanzi, R., Giannini, E., Colucci, M. A., Mignogna, G., Barra, D., et al. (2005). Biological activities of Bv8 analogues. Br. J. Pharmacol. 146 (5), 625-632. doi:10.1038/sj.bjp.0706376

Negri, L., Lattanzi, R., Giannini, E., and Melchiorri, P. (2007). Bv8/Prokineticin proteins and their Receptors. Life Sci. 81 (14), 1103-1116. doi:10.1016/j.lfs. 2007.08.011

Negri, L., and Maftei, D. (2018). Targeting the prokineticin system to control chronic pain and inflammation. Curr. Med. Chem. 25 (32), 3883-3894. doi:10. 2174/0929867324666170713102514

Ng, K. L., Li, J. D., Cheng, M. Y., Leslie, F. M., Lee, A. G., and Zhou, Q. Y. (2005). Dependence of olfactory bulb neurogenesis on prokineticin 2 signaling. Science 308 (5730), 1923-1927. doi:10.1126/science.1112103

Rubio-Perez, J. M., and Morillas-Ruiz, J. M. (2012). A review: inflammatory process in Alzheimer's disease, role of cytokines. ScientificWorldJournal 2012, 756357-756372. doi:10.1100/2012/756357

Sawe, N., Steinberg, G., and Zhao, H. (2008). Dual roles of the MAPK/ERK1/2 cell signaling pathway after stroke. J. Neurosci. Res. 86 (8), 1659-1669. doi:10.1002/ jnr.21604

Schirinzi, T., Maftei, D., Pieri, M., Bernardini, S., NicolaMercuri, B. N. B., Lattanzi, R., et al. (2021). Increase of prokineticin-2 in serum of patients with Parkinson's disease. Mov Disord. doi:10.1002/mds.28458

Schweitz, H., Pacaud, P., Diochot, S., Moinier, D., and Lazdunski, M. (1999). MIT(1), a black mamba toxin with a new and highly potent activity on intestinal contraction. FEBS Lett. 461 (3), 183-188. doi:10.1016/s0014-5793(99)01459-3

Severini, C., Lattanzi, R., Maftei, D., Marconi, V., Ciotti, M. T., Petrocchi Passeri, P., et al. (2015). Bv8/prokineticin 2 is involved in A $\beta$-induced neurotoxicity. Sci. Rep. 5, 15301. doi:10.1038/srep15301

Severini, C., Passeri, P. P., Ciotti, M., Florenzano, F., Possenti, R., Zona, C., et al. (2013). Bindarit, inhibitor of CCL2 synthesis, protects neurons against amyloid- $\beta$-induced toxicity. J. Alzheimers Dis. 38 (2), 281-293. doi:10. 3233/JAD-131070

Soga, T., Matsumoto, S. I., Oda, T., Saito, T., Hiyama, H., Takasaki, J., et al. (2002). Molecular cloning and characterization of prokineticin receptors. Biochim. Biophys. Acta 1579 (2-3), 173-179. doi:10.1016/s0167-4781(02)00546-8

Su, G., Sun, G., Liu, H., Shu, L., Zhang, W., and Liang, Z. (2020). Prokineticin 2 relieves hypoxia/reoxygenation-induced injury through activation of Akt/ mTOR pathway in H9c2 cardiomyocytes. Artif. Cell Nanomed. Biotechnol. 48 (1), 345-352. doi:10.1080/21691401.2019.1709850

Urayama, K., Guilini, C., Messaddeq, N., Hu, K., Steenman, M., Kurose, H., et al. (2007). The prokineticin receptor-1 (GPR73) promotes cardiomyocyte survival and angiogenesis. FASEB J. 21 (11), 2980-2993. doi:10.1096/fj.07-8116com 
Urayama, K., Guilini, C., Turkeri, G., Takir, S., Kurose, H., Messaddeq, N., et al. (2008). Prokineticin receptor-1 induces neovascularization and epicardialderived progenitor cell differentiation. Arterioscler Thromb. Vasc. Biol. 28 (5), 841-849. doi:10.1161/ATVBAHA.108.162404

Yang, Z., Wu, Y., Wang, L., Qiu, P., Zha, W., and Yu, W. (2020). Prokineticin 2 (PK2) rescues cardiomyocytes from high glucose/high palmitic acid-induced damage by regulating the AKT/GSK $3 \beta$ pathway in vitro. Oxid. Med. Cell. Longev. 18, 2020. doi:10.1155/2020/3163629

Yao, H. H., Ding, J. H., Zhou, F., Wang, F., Hu, L. F., Sun, T., et al. (2005). Enhancement of glutamate uptake mediates the neuroprotection exerted by activating group II or III metabotropic glutamate receptors on astrocytes. J. Neurochem. 92 (4), 948-961. doi:10.1111/j.1471-4159. 2004.02937.x

Zhang, C., Truong, K. K., and Zhou, Q. Y. (2009). Efferent projections of prokineticin 2 expressing neurons in the mouse suprachiasmatic nucleus. PLoS One 4 (9), e7151. doi:10.1371/journal.pone.0007151

Zhao, H., Sapolsky, R. M., and Steinberg, G. K. (2006). Phosphoinositide-3-kinase/ akt survival signal pathways are implicated in neuronal survival after stroke. Mol. Neurobiol. 34 (3), 249-270. doi:10.1385/MN:34:3:249
Zhao, H., Shimohata, T., Wang, J. Q., Sun, G., Schaal, D. W., Sapolsky, R. M., et al. (2005). Akt contributes to neuroprotection by hypothermia against cerebral ischemia in rats. J. Neurosci. 25 (42), 9794-9806. doi:10.1523/JNEUROSCI. 3163-05.2005

Zuena, A. R., Casolini, P., Lattanzi, R., and Maftei, D. (2019). Chemokines in alzheimer's disease: new insights into prokineticins, chemokine-like proteins. Front. Pharmacol. 10, 622. doi:10.3389/fphar.2019.00622

Conflict of Interest: The authors declare that the research was conducted in the absence of any commercial or financial relationships that could be construed as a potential conflict of interest.

Copyright (C) 2021 Lattanzi, Severini, Maftei, Saso and Badiani. This is an openaccess article distributed under the terms of the Creative Commons Attribution License (CC BY). The use, distribution or reproduction in other forums is permitted, provided the original author(s) and the copyright owner(s) are credited and that the original publication in this journal is cited, in accordance with accepted academic practice. No use, distribution or reproduction is permitted which does not comply with these terms. 